\title{
LA EDUCACIÓN AMBIENTAL EN LA FORMACIÓN INICIAL DEL PROFESIONAL UNIVERSITARIO
}

\author{
Environmental Education in the Initial Training of the University Professional
}

Guillermo Pablo Leyva Leyva, MsC Universidad de Oriente, Cuba https://orcid.org/0000-0001-6709-5452 gleyva@uo.edu.cu

\author{
Ricardo Mancebo Ayala, MsC \\ Universidad de Oriente, Cuba \\ https://orcid.org/0000-0002- 9594-2105 \\ r.mancebo@uo.edu.cu
}

\author{
Yuleisi Ochoa Nápoles, MsC. \\ Universidad de Oriente, Cuba \\ https://orcid.org/0000-0002-6106-5225 \\ yuli.ochoa@uo.edu.cu
}

Palabras claves: Educación Ambiental, Gestión Ambiental, Problemas Ambientales.

Keywords: Environmental Education, Environmental Management, Environmental Problems.
Recibido: 18 de septiembre de 2020

Aceptado: 11 de diciembre de 2020

\section{RESUMEN}

La formación de profesionales de la educación superior en Cuba, parte desde su concepción curricular a dotarlos de una serie de elementos de carácter técnico, metodológico y didáctico hacia una concepción de educación ambiental, teniendo en cuenta las estrategias educativas y el trabajo sistemático en la labor docente metodológica y científico investigativa, el tratamiento a los objetivos de la agenda 2030 y la Tarea Vida, del cual se estructuran las acciones fundamentales para lograr formar en ellos valores atemperados a las directrices que el gobierno y el ministerio de ciencia, tecnología y medio ambiente que junto a la academia de ciencias de Cuba definen para ello en pos de la sostenibilidad del país.

\section{ABSTRACT}

The formation of professionals of higher education in Cuba, starts from its curricular conception to endow them with a series of technical, methodological and didactic elements towards a conception of environmental education, taking into account the educational strategies and the systematic work in the work methodological and scientific research professor, the treatment of the objectives of the 2030 agenda and the life task, of which the fundamental actions are structured to achieve the formation in them of values tempered by the directives of the government and the Ministry of Science, Technology and the Environment that together with the academy of sciences of Cuba they define for it for the sustainability of the country. 


\section{INTRODUCCIÓN}

Cuando leemos y escuchamos acerca del medio ambiente, de sus problemáticas y conflictos, tanto a nivel local como global, es imprescindible, tener los conocimientos básicos acerca del tema, en el accionar ciudadano y como profesionales, independientemente de la ciencia que se estudia. La introducción de conceptos: medio ambiente, ciencias ambientales, educación ambiental, entre muchos otros relacionados con el tema, son imprescindibles en su vinculación con las carreras universitarias.

Se entiende el medio ambiente como ámbito de vida, desde una perspectiva holística, entendiendo que su cuidado y conservación asegura el bienestar y la calidad de vida futura de nuestras descendencias, entenderíamos entonces la importancia de estudiar estos conceptos e integrarlos a la vida diaria y a la profesión, con acciones conscientes que den cumplimiento a tales argumentos, ya que es necesario contar con teorías y prácticas que ayuden a entender los procesos de mundo social y natural, y en tal sentido es el objetivo esencial: brindar nociones y herramientas básicas para comprender el ambiente desde una perspectiva compleja y holística.

Por lo que es deber accionar desde cada una de las ciencias para construir un concepto de Educación Ambiental que tenga en cuenta la relación sociedad-naturaleza-educación-economía, así como ambiente y ecología, problemas ambientales, desarrollo sostenible, derecho ambiental, gestión ambiental, entre otros temas que contribuyen entre el accionar profesional y el medio ambiente, con enfoques, experiencias e instrumentos que puedan ser abordados.

A partir del presente trabajo hay que repensar y construir juntos nuevas ideas, estrategias y alternativas en pos de un futuro más amigable con nuestro entorno, es decir, con nuestra casa de la cual todos formamos parte desde distintos roles, acciones que realizamos en el día a día, en una aproximación para estimular y fomentar a todos los interesados en el tema ambiental a "accionar" para lograr construir un futuro mejor, en la cual se promuevan valores de compromiso, responsabilidad, participación; y sobre todo, la reflexión-acción de cómo transitar por este camino, donde la sostenibilidad se convierte en la meta a dejar a las futuras generaciones para poder transformar la conducta de los individuos en un hábitat mejor.

\section{DESARROLLO}

El mundo de hoy se encuentra en un proceso hacia la globalización, teniendo como tendencias: la sostenibilidad, en el marco de las nuevas políticas de desarrollo local, regional y global, estas pautas, requieren de un cambio de valores de la sociedad, donde se prioricen la calidad de vida a los factores económicos y la transformación e innovación de la tecnología para solucionar los problemas ambientales existentes y su prevención de futura.

La participación de la comunidad universitaria, la responsabilidad social y los cambios en los sistemas educativos y de formación de los recursos humanos, son fundamentales para estar preparados ante los nuevos desafíos que debemos de enfrentar y del cual las universidades tienen la responsabilidad de ser parte activa de ese cambio.

La Educación Ambiental, fue definida como instrumento para alcanzar el Desarrollo Sostenible en la Conferencia de la Tierra en Río de Janeiro (Brasil) 1992, y dónde se presentó a la educación superior como medio para poder alcanzarlo, ya en el año 2005, Naciones Unidas declara la "Década de las Naciones Unidas de la Educación para el Desarrollo Sostenible" que fuera establecida con el objetivo de contribuir a la formación y concientización de la sociedad en los problemas socio ambientales a los que se enfrenta hoy la humanidad.

En ese contexto, dando cumplimento además a lo establecido en la constitución recién aprobada en 2018 por la asamblea nacional del poder popular y prevista para su referendo popular el $24 / 02 / 2019$, donde se establece que la república promueve la protección y conservación del medio ambiente y el enfrentamiento al cambio climático; en tanto, se estructura la obligatoriedad de la Educación Ambiental, debiéndose crear mecanismos internos desde todas las universidades para lograr un accionar común en las estrategias educativas y formativas.

El propósito fundamental que se plantea a la educación ambiental, desde el proceso formativo universitario, es lograr que los estudiantes comprendan la naturaleza compleja del medio ambiente (resultante de la interacción de sus diferentes aspectos: físicos, biológicos, sociales, culturales, económicos, financieros, etc.) y adquieran los conocimientos necesarios para poder actuar concienzudamente a cambios importantes en el desarrollo social desde lo local a lo global.

Dentro de los principales objetivos de la educación ambiental está: conocer la labor de las principales organizaciones gubernamentales y no gubernamentales, nacionales e internacionales, comprometidas con la problemática ambiental 
mundial. Distinguir las causas que alteran el ambiente e identificar la interacción entre los factores naturales y la intervención humana.

En tanto, la educación ambiental es un proceso de formación que permite la toma de conciencia de la importancia del medio ambiente, que promueve en la ciudadanía el desarrollo de valores y nuevas actitudes que contribuyan al uso racional de los recursos naturales y a la solución de los problemas ambientales que enfrentamos en nuestro entorno.

Para ello, debemos de utilizar recursos innovadores en las ciencias pedagógicas, las ciencias naturales y sociales; se parte de un conocimiento crítico que busca la trasformación y la construcción de una sociedad más sustentable, equitativa y participativa, por lo tanto, la educación ambiental es una filosofía de vida y de los derechos humanos.

Los principales objetivos de la educación ambiental, van dirigidos a conocer la labor de las principales organizaciones gubernamentales y no gubernamentales, nacionales e internacionales, comprometidas con la problemática ambiental local, nacional y mundial; además, de analizar las causas que alteran el ambiente, identificar la interacción entre los factores naturales y la intervención humana, entre otros aspectos esenciales.

Por lo tanto, Educación ambiental, proceso continuo y permanente, que constituye una dimensión de la educación integral de todos los ciudadanos, orientada a que en la adquisición de conocimientos, desarrollo de hábitos, habilidades, capacidades y actitudes y en la formación de valores armonicen las relaciones entre los seres humanos, y de ellos con el resto de la sociedad y la naturaleza, para propiciar la orientación de los procesos económicos, sociales y culturales hacia el desarrollo sostenible.

La cultura ambiental es la forma de como los seres humanos se relacionan con el medio ambiente, y para comprenderla se debe comenzar por el estudio de los valores; estos, a su vez, determinan las creencias y las actitudes y, finalmente, todos son elementos que dan sentido al comportamiento ambiental.

Por lo tanto, los medios de difusión masiva tendrán la responsabilidad de incorporar en el diseño y ejecución de su programación televisiva, radial y en la prensa plana, los temas ambientales que propicien una mayor información y conocimiento por la población, de las complejas interrelaciones y vínculos entre los procesos de desarrollo económico y social con la protección del medio ambiente, propiciando aumentar la cultura ambiental de la ciudadanía.

Se necesita de una educación ambiental para la sustentabilidad con una nueva ética que abarque a todos los seres vivos para que las sociedades humanas vivan en armonía con la naturaleza, de la que dependen para su supervivencia y bienestar. El objetivo a largo plazo de la educación ambiental es fomentar actitudes y comportamientos compatibles con esta nueva ética, que favorezca la gestión ambiental.

La gestión ambiental es un conjunto de actividades, mecanismos, acciones e instrumentos, dirigidos a garantizar la administración y uso racional de los recursos naturales mediante la conservación, mejoramiento, rehabilitación y monitoreo del medio ambiente y el control de la actividad del hombre en esta esfera. La gestión ambiental aplica la política ambiental establecida mediante un enfoque multidisciplinario, teniendo en cuenta el acervo cultural, la experiencia nacional acumulada y la participación ciudadana.

Asimismo, los problemas ambientales prácticamente afectan a la totalidad de los elementos de la naturaleza: el agua, el suelo, la capa vegetal, los animales y el clima. Un problema ambiental es cualquier alteración que provoca desequilibrio en un ambiente dado, afectándolo negativamente, para que cumpla los propósitos que este tiene en cada ecosistema, para desarrollar su medio ambiente y el desarrollo sostenible.

Entiéndase el medio ambiente como: sistema de elementos abióticos, bióticos y socioeconómicos con que interactúa el hombre, a la vez que se adapta al mismo, lo transforma y lo utiliza para satisfacer sus necesidades, y el desarrollo sostenible, proceso de elevación sostenida y equitativa de la calidad de vida de las personas, mediante el cual se procura el crecimiento económico y el mejoramiento social, en una combinación armónica con la protección del medio ambiente, de modo que se satisfacen las necesidades de las actuales generaciones, sin poner en riesgo la satisfacción de las necesidades de las generaciones futuras.

Se puede identificar como problemas de medio ambiente: desde la globalización y la industrialización, el ser humano ha repercutido enormemente en el cambio climático y en el impacto ambiental sin duda todos estos productos químicos que se utilizan en el proceso de materias provocan contaminación que afectan la capa de ozono, los mares, el aire, las aguas entre otros.

Dentro de los principales problemas ambientales del planeta encontramos: el cambio climático, la destrucción de la capa de ozono, la deforestación, la contaminación del agua y su escasez, el dióxido de carbono, acidificación y la degradación y/o erosión del suelo, hidrocarburos clorados y otras causas de contaminación como el derramamiento de 
petróleo están destruyendo los ecosistemas marinos de nuestro planeta, la extinción de especies y pérdida de biodiversidad, la invasión y tráfico ilegal de especies, pero la "causa que produce las demás causas", es la acción indiscriminadamente del ser humano, por acciones inescrupulosas y por falta de conciencia ambiental para la supervivencia humana.

Dentro de las principales consecuencias de la contaminación encontramos: la contaminación en las plantas, que se han visto afectadas e incluso hay especies que han desaparecido por la polución desde el punto de vista ecológico, también la escasez de lluvias y la contaminación de agua, aire y suelo han tenido un papel muy importante, que traen como efectos negativos el riesgo de padecer enfermedades respiratorias agudas, como la neumonía, y crónicas, como el cáncer del pulmón y las enfermedades cardiovasculares, entre otras.

Dentro de los principales objetivos y metas que se proponen acerca de la educación ambiental están:

1. Lograr que las personas naturales y jurídicas comprendan la complejidad del ambiente natural y el creado por el hombre para que adquieran los conocimientos, valores, actitudes y habilidades prácticas que les permitan participar de manera responsable y efectiva en la previsión y resolución de los problemas ambientales.

2. Adquirir hábitos y costumbres acordes con una apropiación cuidadosa de los recursos de uso cotidiano y los medios de transporte.

3. Conocer la labor de las principales organizaciones gubernamentales y no gubernamentales, nacionales e internacionales, comprometidas con la problemática ambiental, para identificar las causas que alteran el ambiente y la interacción entre los factores naturales y la intervención humana.

4. Reconocer la importancia del impacto que ejercen los diferentes modelos económicos en el ambiente desde lo universal y lo particular en el país.

5. Identificar las formas de apropiación de los recursos naturales y el impacto ambiental que las mismas generan.

6. Mostrar claramente la interdependencia económica, política y ecológica del mundo moderno, debido a la cual las decisiones y las acciones de diferentes países pueden tener repercusiones internacionales.

7. Desarrollar a través de la educación una conciencia ética hacia los valores ambientales, ya que cuando se carece de un pensamiento ético-ambiental no se asumen actitudes de respeto; así lo muestran las actividades humanas que conducen a la degradación ambiental.

8. Proporcionar la información y los conocimientos necesarios en la población para que ésta adquiera conciencia de los problemas del ambiente, creando en ella una disposición, motivación, sentido de responsabilidad y compromiso para trabajar individual y colectivamente en la búsqueda de soluciones.

9. Promover una clara conciencia acerca de la interdependencia económica, social, política y ecológica en áreas urbanas y rurales.

10. Dar a cada persona las oportunidades para que adquiera los conocimientos, valores, actitudes, compromisos y habilidades necesarios para proteger y mejorar el ambiente y con ello alcanzar los objetivos de desarrollo sustentable.

11. Actuar con una visión socio-ambiental: incorporando en el diseño y operación de sus actividades, la reflexión y discusión sobre las actividades a desarrollar, con una ética personal y social, promoviendo cambios en los hábitos y costumbres personales cotidianos, e incidiendo en los procesos sociales, hacia una educación integral crítica, donde los procesos educativos no deben restringirse a la transmisión de información y a crear acerbo de conocimientos, sino al desarrollo de habilidades técnicas y de razonamiento; asumiendo una actitud crítica frente a los comportamientos y valores personal y colectivo.

12. Promocionar, desde lo pedagógico, una comprensión integral de las características de medio natural y del medio transformado por el ser humano, a través de las estrategias educativas y su medición, donde se incorpora la dimensión ambiental y el desarrollo económico y financiero desde lo local, en los diversos niveles, modalidades y áreas del conocimiento, favoreciendo la adquisición de conocimientos, valores, comportamientos, destrezas y prácticas cotidianas, para la toma de conciencia sobre la importancia del medio ambiente y su desarrollo, para crear las condiciones para facilitar una clara comprensión de la interdependencia económica política y ecológica.

Dentro de los principios básicos de la educación ambiental, se plantean los siguientes:

1. Considerar al ambiente en forma integral, es decir, lo natural y lo construido (lo tecnológico, social, económico, político, moral, cultural, histórico y estético).

2. Asumir un enfoque interdisciplinario para el tratamiento de la dimensión ambiental, que posibilite una perspectiva holística y equilibrada.

3. Tratar la temática ambiental desde lo particular a lo general tiene como finalidad que los estudiantes se formen una idea de las condiciones ambientales de otras áreas, que identifiquen las condiciones que prevalecen en 
las distintas regiones geográficas y políticas, además de que reflexionen sobre las dimensiones mundiales del problema ambiental para que los sujetos sociales se involucren en los diferentes niveles de participación y responsabilidad.

4. Complejidad de los problemas ambientales, por lo cual es necesario desarrollar el pensamiento crítico y las habilidades para resolverlos.

5. Promover el conocimiento, la habilidad para solucionar problemas, la clasificación de valores, la intervención y la evaluación de situaciones, en los estudiantes en formación, cuyo interés especial sea la sensibilización ambiental para aprender sobre la propia comunidad.

6. Capacitar a los alumnos para que desempeñen un papel en la planificación de sus experiencias de aprendizaje y darles la oportunidad de tomar decisiones.

7. Evaluar las implicaciones ambientales en proyectos de los grupos estudiantiles de desarrollo.

8. Insistir en la necesidad de cooperación a todos los niveles para la prevención y la solución de los problemas ambientales.

El conocimiento de los problemas ambientales, puede, teniendo como basamento estos objetivos y principios planteados en este trabajo, ayudar a comprender un aún más la complejidad de la realidad que vivimos. Esto no significa, que los contenidos por sí solos conduzcan al estudiante a un cambio de actitudes hay que intencionar su estudio y darle tratamiento a través de todas las asignaturas y con el diseño e implementación de estrategias de Educación ambiental que trasversalicen toda la formación del profesional universitario desde un enfoque profesional investigativo y preventivo. En este sentido, se propone promover una educación ambiental que permita cuestionar los actuales modelos de desarrollo, pues estos son los responsables del deterioro ecológico y social que viven los países subdesarrollados, el cual es diferente al que se presenta en otros países.

\section{CONCLUSIÓN}

Entre los objetivos básicos de la educación ambiental que tienen vigencia, se encuentran: la toma de conciencia y la sensibilización de los individuos y grupos sociales acerca del entorno global y su problemática, de los conocimientos para comprender el entorno global, su problemática, la presencia del hombre en el entorno, la responsabilidad y el papel crítico que lo atañen, la actitud para adquirir valores sociales, a interesarse por el medio ambiente, a tener una motivación fuerte, y las actitudes y aptitudes necesarias para querer participar en la protección del medio ambiente y mejorarlo, la capacidad de evaluación de las medidas y los programas de educación ambiental en función de los factores ecológicos, políticos, económicos, sociales, estéticos y educativos y la participación para garantizar las medidas para resolver los problemas del medio ambiente.

En el logro de tal empeño desempeñan un papel primordial las universidades las que, basadas en los objetivos y principios básicos de la educación ambiental expuestos, deben trazarse metas, estrategias, formas y vías de instrumentación desde el currículo y extracurricularmente.

\section{BIBLIOGRAFÍA}

1. 21 Conferencia de Química: 3 al 5 de diciembre de 2014: http://ciq.e Padecimiento de los Hombres y mujeres se relacionan con la calidad de agua que consumen.

2. Bárcena, Alicia y Prado, A. 2016. Agenda 2030 y los Objetivos de Desarrollo Sostenible Una oportunidad para América Latina y el Caribe (CEPAL). Naciones Unidas.

3. Congreso Internacional de Desarrollo Local 4 al 7 de noviembre de 2014: http://eventos.uo.edu.cu/ $p=417$ Nuestro evento internacional de mayor experiencia:

4. Garrido Vázquez, Raúl J. 2003. Estudio de caso: Cuba. Aplicación de instrumentos económicos en la política y la gestión ambiental. Naciones Unidas. Santiago de Chile.

5. Gustavo Wilches-Chaux. 1995. Desastres y el medio ambiente. PNUD. 2da Edición.

6. Ley 81 del medio ambiente del 11 de julio de 1997, de la República de Cuba.

7. Pérez, S.; Guillén, L. y Heredia, A. (2017) La formación humanista. Un reto ante el desarrollo de las tecnologías de la información y las comunicaciones http://ojs.formacion.edu.ec/index.php/rif/article/view/67 DOI: https://doi.org/10.34070/rif.v5i2 